\title{
PHYSICS OF NEUTRAL-TO-IONIC PHASE TRANSITION IN ORGANIC CHARGE TRANSFER SEMICONDUCTING COMPOUNDS
}

\author{
H. Cailleau, M.H. lemée-Cailleau, M. le Cointe \\ Groupe Matière Condensée et Matériaux, UMR CNRS 6626 \\ I \\ Université de Rennes 1, 35042 Rennes cedex, France \\ AND T. LUTY \\ Institute of Physical and Theoretical Chemistry, Technical University of Wrocław \\ Wybrzeże Wyspiańskiego 27, 50-370 Wrocław, Poland
}

An uncommon excitonic instability takes place in some exotic semiconducting compounds. Indeed, the equilibrium neutral-to-ionic (N-I) phase transition, as well as the non-equilibrium photo-induced phase transformation, observed in some organic charge-transfer complexes, originate from intra- and inter-chain cooperative effects between structurally relaxed charge-transfer excitations. This electronic-structural phase transition manifests itself by a change of the degree of charge-transfer and a dimerization distortion with the formation of donor-acceptor pairs along the stacking axis in the I phase. Thermal charge-transfer excitations associated with the formation of I strings along $N$ chains are at the heart of the mechanism of this phase transition. These relaxed electronic excitations, which are an intrinsic feature of low-dimensional systems with strong electron-phonon coupling, can be described in terms of self-trapping and self-multiplication of charge-transfer excitons. Precise structural studies on the prototype compound, tetrathiafulvalene-p-chloranil allow to highlight the respective role taken by the ionicity and the dimerization. Symmetry and thermodynamics analysis of the N-I transition, based on recent determination of the pressure-temperature phase diagram, make possible to present a consistent picture of this phase transition. Supported by theoretical considerations taking into account the interplay between quantum and thermal effects, the experimental observations show that the N-I transition results from the condensation and the ordering (crystallization) of charge-transfer excitations, following a phase diagram analogous to the solid-liquid-gas one.

PACS numbers: $61.50 . K s, 71.35 . A a, 71.38 .+i$ 


\section{Introduction}

Excitonic instabilities, such as electron-hole droplet condensation or possible Bose condensation, are known to occur in some photo-excited semiconductors [1]. Another kind of excitonic instability related to the condensation of self-trapped charge-transfer (CT) excitons appears in some exotic semiconducting compounds [2]. Thus, different quasi-one-dimensional organic compounds exhibit photo-induced phase transformations associated with coupled electronic and structural changes of molecular identity, such as valence state or ionicity [3]. Due to their intrinsic low-dimensionality and to strong electron-phonon coupling, the structurally relaxed photo-excited states involve a large number of molecules and atoms along the chains. These specific relaxed excitations can be described in terms of self-multiplication of self-trapped CT excitons $[2,3]$. This situation is carried to extremes in the case of the neutral-to-ionic (N-I) transition where the interchain cooperativity drives at thermal equilibrium an electronic-structural phase transition. This leads not only to three-dimensional condensation of self-trapped CT excitons, but also to their ordering (crystallization) [4-6]. It results in fascinating new liquid-like and solid-like states of condensed electronic excitations. The study of the physics of the N-I transition allows to address basic questions concerning both the cooperative effects between self-trapped excitons and the so-called molecular multistability in the field of molecular electronics.

This uncommon phase transition, which takes place in some organic mixed-stack CT complexes presenting small optical CT gap, has been discovered by the observation of a drastic change of the visible absorption spectra [7]. Contrary to segregated-stack CT crystals which form the well-known family of one-dimensional conductors, in mixed-stack CT crystals electron-donor (D) and electron-acceptor (A) molecules alternate along the same stack. Due to this alternance they are always non-metallic and at best small-gap semiconductors. The N-I transition manifests itself by a change of the degree of CT between $\mathrm{D}$ and $\mathrm{A}$ and a dimerization distortion with the formation of $\left(\mathrm{D}^{+} \mathrm{A}^{-}\right)$dimers along the stacking axis in the I phase

$$
\begin{array}{ll}
N \text { phase : } & \ldots D^{0} A^{0} D^{0} A^{0} D^{0} A^{0} D^{0} A^{0} D^{0} A^{0} D^{0} A^{0} \ldots \\
\text { I phase : } & \ldots\left(D^{+} A^{-}\right)\left(D^{+} A^{-}\right)\left(D^{+} A^{-}\right)\left(D^{+} A^{-}\right)\left(D^{+} A^{-}\right)\left(D^{+} A^{-}\right) \ldots
\end{array}
$$

As in other low-dimensional systems the ground state results from a delicate balance between electron-transfer energy, electron-electron and electron-lattice interactions. Hybridization of HOMO and LUMO orbitals of the D and A molecules respectively, due to a non-zero transfer integral especially along the chain, leads to a partial degree of CT in the ground state, $q(0<q<1)$. When the difference between the ground state energies of the quasi- $\mathrm{N}$ phase and the quasi-I phase is of the order of thermal energy, an equilibrium N-I phase transition can occur. Most likely, the phase transition can be related to the condensation of CT excitations. Thus, a self-multiplication of self-trapped CT excitons [2] along $N$ chains results from strong attractive Coulomb interactions between $\left(\mathrm{D}^{+} \mathrm{A}^{-}\right)$dimers and leads to the formation of $I$ strings composed of several adjacent dimers

$$
\ldots D^{0} A^{0} D^{0} A^{0} D^{0} A^{0}\left(D^{+} A^{-}\right)\left(D^{+} A^{-}\right)\left(D^{+} A^{-}\right)\left(D^{+} A^{-}\right)\left(D^{+} A^{-}\right) D^{0} A^{0} D^{0} A^{0} D^{0} A^{0} \ldots
$$


These structurally relaxed electronic excitations have first been discussed by $\mathrm{Na}$ gaosa [8] in relation to the intrinsic bipolaronic state of one-dimensional systems. Unlike conducting polymers or mixed-valence $\mathrm{M}-\mathrm{X}$ chains, $\mathrm{CT}$ excitations exhibit here interchain cooperativity. This leads to the growing and the three-dimensional ordering of I strings, and so can drive an equilibrium phase transition. The understanding of intra- and inter-chain cooperative effects is also crucial to explain non-equilibrium phenomena such as photo-induced phase transformations [9] or switching between low- and high-electric conductivity states under high-electric field [10].

The aim of this paper is to overview the physics of the N-I phase transition, especially the nature of the cooperative mechanism. The discussion of the physical features is based on recent structural studies performed by our group and results on electronic properties obtained by Japanese's groups on the prototype compound, tetrathiafulvalene-p-chloranil (TTF-CA). This allows to present a consistent picture of this uncommon electronic-structural phase transition.

\section{Symmetry analysis and electronic-structural changes}

It is well known from phenomenological Landau theory of phase transitions that every density of probability (electronic, atomic position...) describing the two phases can be expressed as $\rho=\rho_{0}+\Delta \rho$, where $\rho_{0}$ is totally symmetric with respect to the high-symmetry phase and $\Delta \rho$ describes the symmetry lowering arising in the low-symmetry phase. This term, non-zero only in the low-symmetry phase, is proportional to a symmetry-breaking order parameter $\eta$. In general, structural phase transitions are associated with a change of space group without drastic change of $\rho_{0}$. A typical example is given by a displacive ferroelectric phase transition where $\eta$ is the relative displacement of ionic sublattices. There are fewer examples of isostructural phase transitions (metal-insulator Mott transition, spin transition...), exhibiting a discontinuous change of $\rho_{0}$, but without change of space group (no symmetry-breaking). In the last case $\rho_{0}$ plays the role of a totally symmetric order parameter, in the same sense as the density for the liquid-gas transition, and it is non-zero in both two phases. From a symmetry point of view there is no difference in nature between the two phases and the transition line finishes at a critical point. The N-I phase transition is unusual because it involves, on an equal footing, the two types of order parameter, associated with the ionicity change and the dimerization. The terms "ionicity change" and "dimerization" are used as generic expressions which represent all electronic and structural changes (intra- and inter-molecular) corresponding to the part preserving or not the symmetry, respectively.

In TTF-CA, at atmospheric pressure and room temperature, the $\mathrm{N}$ phase is stable. The space group is $P 2_{1} / n$ with two symmetry related undimerized pairs [11]. The TTF(D) and CA(A) molecules are located on sites with a center of inversion and form alternating stacks along the crystallographic $a$ axis. The degree of CT $q_{\mathrm{N}}$ is about 0.3 , as determined by electronic and vibrational spectroscopies [12]. At $T_{\mathrm{N}-\mathrm{I}}=81 \mathrm{~K}$, after a strongly first order transition, the system becomes more ionic: the degree of CT increases to about 0.7 . There is no change of the multiplicity of the unit cell and the symmetry lowering is shown on the diffrac- 


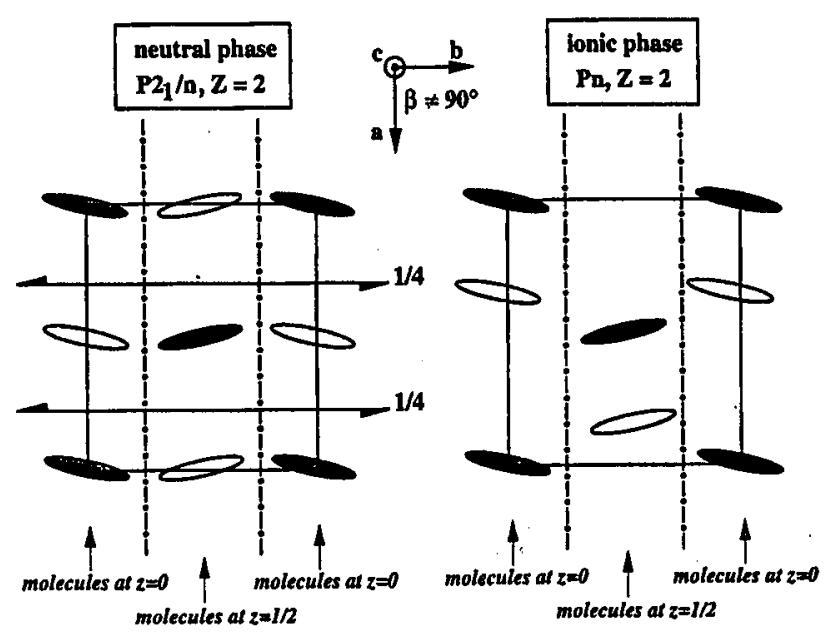

Fig. 1. Schematic drawing of the symmetry lowering at the N-I transition in TTF-CA.

tion pattern only by the appearance of weak $0 k 0: k=2 n+1$ reflections indicating the loss of the screw axis [13]. Thus, with a symmetry-breaking order parameter of $B_{\mathrm{u}}$ symmetry, the space group of the I phase is $P n$ with two equivalent dimers related by the glide plane (Fig. 1). This implies a ferroelectric arrangement between the dimerized chains with a polarization vector in the $(a, c)$ plane. Instead of the regular $\mathrm{D}-\mathrm{A}$ distance along the stack in the $\mathrm{N}$ phase, an alternation of intradimer and interdimer distances appears and can represent $\eta$. The intensity of the new reflections which indicate the symmetry breaking is proportional to $\eta^{2}$. For its part the totally symmetric change is characterized by an abrupt decrease at the N-I transition of $b$ and $c$ unit cell parameters, in conjunction with the enhancement of the interstack coupling due to the increase in CT.

The molecular deformation is directly related to both the ionicity change which induces a centro-symmetric distortion and the dimerization which causes the loss of the inversion symmetry [5]. The changes in geometry of CA molecule are closely correlated with ${ }^{35} \mathrm{Cl}$ nuclear quadrupole resonance (NQR) results [14]: the ionicity change leads to a large frequency shift between lines of $\mathrm{N}$ and I phases, while the dimerization induces a splitting of the I lines. Since the frequency shift for each chlorine nucleus is proportional in a first approximation to the local distortion, this splitting is proportional to $\eta$. The lifetime of transient structurally relaxed $\mathrm{CT}$ excitations in the $\mathrm{N}$ phase, as measured by photo-induced experiments, is in the nanosecond range [9]. Thus, contrary to electronic and vibrational optical spectroscopies which probe the instantaneous degree of CT $q_{\mathrm{I}}$ and $q_{\mathrm{N}}$ of specific molecules (possibly coexistence of two lines), NQR probes the dynamical average between different states of the degree of CT (only one average line), i.e. the true thermodynamic parameter. 


\section{Phase diagram and thermodynamics}

The study of the influence of pressure is a good way to improve our knowledge on the thermodynamics of the N-I transition. The pressure-temperature $(P, T)$ phase diagram of TTF-CA has recently been determined by using neutron diffraction along with NQR measurements under well-controlled hydrostatic pressure (helium pressure cells) [6]. The results obtained from the two techniques are in perfect agreement. The $(P, T)$ phase diagram is presented in Fig. 2. As found in previous studies [15-20], the transition temperature increases under pressure. However, with regard to clamp or diamond-anvil cell, the use of helium pressure cell has allowed the accurate determination of the equilibrium line, especially in the low pressure region.

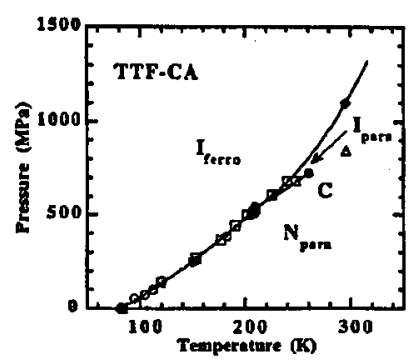

Fig. 2. $(P, T)$ phase diagram: $\diamond$ neutron scattering and $\square$ NQR results [6]. $\diamond$ Vibrational spectroscopy [17-19], $\triangle$ conductivity measurements $[15,16] . C$ is the estimated critical point. $N_{\text {para }}, I_{\text {para }}$ and $I_{\text {ferro }}$ refer to the paraelectrical neutral, paraelectric ionic and ferroelectric ionic phases respectively.

An important feature is that the phase transition becomes less and less discontinuous with increasing pressure, suggesting a critical region at high pressure. First, one can think about the possibility of a tricritical point, beyond which the transition would be of second order, and with reference to the classical picture of two coupled sets of conjugate variables: on the one hand the dimerization order parameter and the electric field, on the other hand the ionicity and the pressure. But the essential observation, clear from both neutron diffraction and NQR, is that the ionicity discontinuity along the transition line extrapolates to zero well before the dimerization one does. This excludes the simple picture of a tricritical point. The N-I first-order transition line must split at high pressure in two lines beyond a triple point: on the one hand a first-order line between a paraelectric $\mathrm{N}$ phase and a paraelectric I phase which is characterized by a jump of ionicity without change of symmetry, on the other hand another first-order line between this paraelectric I phase and the ferroelectric I phase where the dimerization order takes place. The first line, associated to an isostructural phase transition, finishes in a critical point which is probably located, by considering the evolution of ionicity jump, around $250 \mathrm{~K}$ and $700 \mathrm{MPa}$. The second line can become of second order beyond a tricritical point which would be at much higher pressure. This interpretation is corroborated by the observation at room temperature of a maximum of the electric 
conductivity $[15,16]$ at a pressure much below where the dimerization transition occurs [17-19]. This maximum conductivity can be related to a crossover phenomena in the hypercritical regime beyond the critical point. The tendency towards a critical point related to the totally symmetric change is well illustrated by the increase with pressure in the pretransitional evolution of ionicity on each side of $T_{\mathrm{N}-\mathrm{I}}$; this behavior expresses itself in the $b$ cell parameter evolution, as well as in the deviation from the standard thermal evolution of NQR frequencies [6]. On the contrary, no pretransitional effects associated with the symmetry-breaking have been observed in the $\mathrm{N}$ phase, which is consistent with the clear first-order nature of the dimerization transition.

\section{Cooperativity and CT excitations}

Such a phase diagram follows a phenomenological approach describing the $\mathrm{N}-\mathrm{I}$ phase transition in terms of condensation and crystallization of CT excitations. So far the theory of the N-I transition has mainly been focused on the nature of the ground state, either by the study of Peierls-IIubbard-like one-dimensional models [21] or by $a b$ initio calculations [22]. Thermal effects have little been discussed [23, 24]. The present discussion tries to bring out a consistent picture, taking into account the interplay between quantum and thermal effects, as it was recently suggested by one of us [4].

We can represent the ground state energy of a single mixed-stack DA chain as a function of the dimerization parameter (symmetry-breaking intra- and inter-molecular deformation) and the totally symmetric (ionicity) change (cell parameter and centro-symmetric molecular deformation). It presents three minima: one quasi- $\mathrm{N}$ and two equivalent polar quasi-I states (of polarization $\pm P_{0}$ ). For each state, the ground state degree of CT is determined by hybridization of HOMO and LUMO orbitals of the D and A molecules respectively. A distinctive feature of DA chain is that the electron-phonon coupling leading to dimerization may have two origins, modulation of intersite Coulomb interaction (ferroelectric-like instability) and of transfer integral (spin-Peierls-like instability). The role of each contribution is still controversial. This single DA chain is a very typical example of molecular multistability as discussed by Nasu [25] in terms of degenerate or pseudo-degenerate ground states.

A simple picture (Fig. 3) can be drawn in order to build an intuition about this molecular multistability. Thus, the ground state is constructed from two diabatic states as a function of dimerization displacement (for simplicity). The diabatic ground state of an $\mathrm{N}$ chain exhibits a single-well shape centered on zero, while the I one has a double-well shape with two degenerate energy minimum states (I and $\overline{\mathrm{I}}$ ). The adiabatic ground state results from quantum mixing of both configurations due to hybridization. The change of the ground state degree of CT mainly occurs when the two diabatic curves cross. This simple picture allows to visualize the energy difference between non-relaxed states which is given by the optical CT gap (about $0.6 \mathrm{eV}$ for TTF-CA) and the one between structurally relaxed states (of the order of thermal energy). The vibrational spectra, as well as cell parameters, are different for $\mathrm{N}$ and I states, and play a role in their stabilization at finite temperature and pressure. 


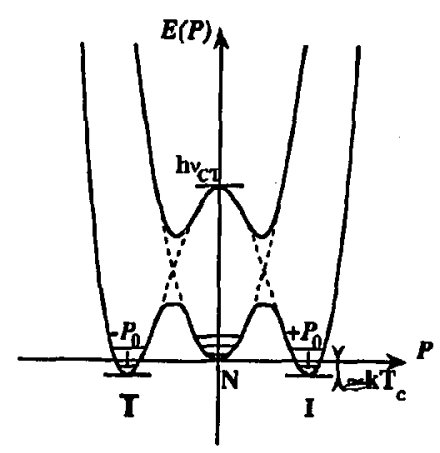

Fig. 3. Schematic drawing of the multistable potential for a DA mixed-stack. The adiabatic ground state and excited state (full lines) are constructed from $\mathrm{N}$ and I diabatic potentials (dashed lines) as a function of polarization.

The nature of the ground state suggests that the dynamics of the system will be of mixed phonon-relaxator character. Thus, pretransitional dynamics which occurs in the $\mathrm{N}$ phase should be governed by two types of excitations. First, a polar vibrational mode of dimerization (ungerade symmetry) may soften and condense

Second, structurally relaxed $\vec{I}$ dimer strings,

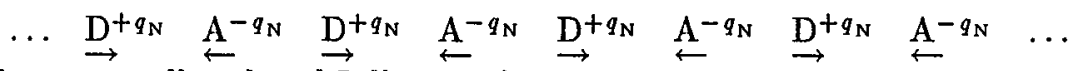

$$
\ldots \mathrm{D}^{+q_{\mathrm{N}}} \mathrm{A}^{-q_{\mathrm{N}}}\left(\mathrm{D}^{+q_{1}} \mathrm{~A}^{-q_{1}}\right)\left(\mathrm{D}^{+q_{1}} \mathrm{~A}^{-q_{1}}\right)\left(\mathrm{D}^{+q_{1}} \mathrm{~A}^{-q_{1}}\right)\left(\mathrm{D}^{+q_{1}} \mathrm{~A}^{-q_{1}}\right) \mathrm{D}^{+q_{\mathrm{N}}} \mathrm{A}^{-q_{\mathrm{N}}} \ldots
$$

which correspond to relaxation-type excitations between the minima in the ground state. The two types of excitations coexist. However, at zero temperature, only the first one is efficient (displacive regime) while, as temperature increases, the relaxed CT excitations become more and more efficient (order-disorder regime).

At zero temperature the DA chain may present a N-I transition driven by pressure when the enthalpies of $I$ and $N$ phases become equal. This phase transition is generally observed as first-order in many theoretical studies of one-dimensional models [21]. The mechanism is displacive with a complete softening of the dimerization mode in the hypothetical case of a continuous N-I transition [26]. At finite temperature thermal CT excitations cause inhomogeneities along the chain. It appears as a mixture of $\mathrm{N}$ species and I dimer strings, and moveable $\mathrm{N}$-I domain-walls allow for thermal mixing of the "compounds". This thermal effect breaks up the long-range dimerization order. In addition, at the border between stable $\mathrm{N}$ and I states, the moveable N-I domain-walls probably dilute the two phases together and a crossover behavior replaces the phase transition. This description is consistent with the well-known statement of Landau on the non-existence of phase transition for one-dimensional systems at finite temperature.

The description of the N-I transition needs to take into account the interchain cooperativity. The three possible states in the stack can be described by a spin-1 Ising variable $S^{z}=\left(P / P_{0}\right)=0, \pm 1$. The total Hamiltonian of the system should include both quadrupolar interchain interactions, $C_{i j}\left(S_{i}^{z}\right)^{2}\left(S_{j}^{z}\right)^{2}$, and polarization interchain interactions, $J_{i j}\left(S_{i}^{z}\right)\left(S_{j}^{z}\right)$, where $i, j$ denote dimers in different stacks. The coupling constants have both electric and elastic origin and it 
is believed that the elastic contribution is quite significant, since in particular the dipolar energy in the ordered phase is weak [13]. This model resembles the spin-1 lattice-gas model, introduced by Blume-Emery-Griffiths for ${ }^{3} \mathrm{He}-{ }^{4} \mathrm{He}$ mixtures [27] and discussed by Lajzerowicz and Sivardière for the condensation and solidification of a simple fluid [28]. The concentration $c=\left\langle\left(S^{z}\right)^{2}\right\rangle$ of the I "compounds" serves as an order parameter for the condensation phase transition, analogous to the density for the gas-liquid transition. Thermal effects average the ionicity and so the average CT is equal to $\langle q\rangle=c q_{\mathrm{I}}+(1-c) q_{\mathrm{N}}$. The polarization interchain interactions break the symmetry of the system and drive the phase transition associated with the three-dimensional ordering of strings of I dimers, characterized by a symmetry-breaking order parameter $\eta=\left\langle S^{z}\right\rangle$.

The experimental results and the model offer the following physical picture for the N-I transition in TTF-CA. In the low-temperature low-pressure regime the DA mixed-stacks are composed of predominantly $\mathrm{N}$ or of predominantly $\mathrm{I}$ molecules and $q_{\mathrm{N}}$ and $q_{\mathrm{I}}$ are substantially different. Condensation of the I strings is accompanied by their ordering in the crystallization (ferroelectric) transition driven by the interstack $J_{i j}$ coupling. Rather small concentration of thermal CT excitations makes the transition discontinuous with no indication of a large disordered state in the $\mathrm{N}$ phase. Actually, only weak precursor effects are indicated in the behavior of NQR frequencies [14], while a small-softening of low-frequency polar lattice-mode is observed by far-infrared spectroscopy [29]. Pressure not only favors the I phase (increase in Madelung energy) [30] but also strongly stimulates quantum mixing (increase in transfer integral [31]) which changes the ground states and makes $\mathrm{N}$ and I "compounds" more and more similar. It is manifested by a convergence of the instantaneous degree of CT with pressure for coexisting $\mathrm{N}$ and I species as observed by near-infrared vibrational spectroscopy at room temperature [19]. An increase in the temperature stimulates disorder between $\mathrm{N}$ and I strings along the stack. With the increase in $T_{N-I}$, the transition becomes less discontinuous showing symptoms of an order-disorder mechanism. Thus a tendency towards a displacive to order-disorder crossover with increasing pressure is observed by Raman scattering [20]. The existence of thermal CT excitations is illustrated by the continuous or quasi-continuous evolution of the thermal average ionicity although a jump clearly remains in the instantaneous degree of CT [19]. The temperature range where they are efficient is particularly visible on NQR results. As long as there is a significant difference in the instantaneous ionicities, it drives a structural transition via interstack coupling and the transition from the $\mathrm{N}$ to the I phase can be viewed as a simultaneous condensation and crystallization of CT excitations. The equilibrium line on the $(P, T)$ phase diagram is like a "sublimation curve", between a disordered "gas" and an ordered "solid" of CT excitations, governed by the interplay of quantum mixing ( $P$ axis) and thermal mixing ( $T$ axis). Both mixings drive the ordering towards a more continuous behavior and the system towards criticality. However, in the upper part of this "sublimation curve" the difference in instantaneous degree of CT diminishes and becomes too small to drive the crystallization transition. The system reaches a triple point first, and the condensation transition to an ionic but disordered (paraelectric) "liquid" 

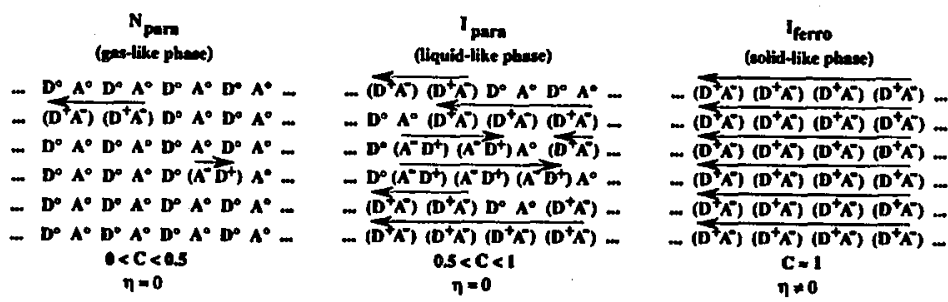

Fig. 4. Schematic view of the different phases: paraelectric neutral (gas-like), paraelectric ionic (liquid-like) and ferroelectric ionic (solid-like).

phase is separated from the crystallization transition of this paraelectric "liquid" to the ordered ferroelectric I phase. A schematic view of these three phases is given in Fig. 4. The condensation line terminates at a critical point where the system reaches its critical thermal average ionicity (the simplest estimation suggests $c_{\mathrm{c}}=1 / 2$ ). In the hypercritical regime along the prolongation of this condensation line one may expect an equal amount of $\mathrm{N}$ and $\mathrm{I}$ species. This is observed at room temperature by electronic optical spectroscopy $[16,19]$ at about $800 \mathrm{MPa}$, and indicated as well by a maximum electric conductivity $[15,16]$. This is also consistent with the interpretation proposed for the maximum electric conductivity observed in the same $(P, T)$ conditions as the signature of the vanishing of the thermal CT gap [16].

Another physical feature is the possibility of unusual ferroelectric properties with simultaneous polar $\mathrm{CT}$ and dimerization fluctuations. The chains are essentially decoupled in the "fluid" phases and so one-dimensional ferroelectric properties [32] can be expected, reflecting I string dynamics. The excitation of an I dimer creates a large dipole moment. We suggest that this is at the origin of the observed large dielectric response along the stack [33]: the divergent increase, on approaching $T_{\mathrm{N}-\mathrm{I}}$, almost independent of frequency, is too large to be attributed to the softening of the polar dimerization mode. In the same way, spin-lattice relaxation rates $T_{1}^{-1}$ in NQR measurements [14] reveal the existence of pretransitional fluctuations with a predominant one-dimensional character. In addition, an anomalous dielectric response associated with a very slow dynamics has been observed and explained in terms of N-I domain walls. However, this interpretation is contradictory with the non-observation of the coexistence of $\mathrm{N}$ and $\mathrm{I}$ lines in the NQR spectra [14]. The possibility of electric conductivity or random-field (defects, impurities) effects cannot be excluded.

Moreover, the entropy variation at $T_{\mathrm{N}-\mathrm{I}}$ close to $k_{\mathrm{B}} \ln 2$ per DA pair has been determined from specific heat measurements on a set of single crystals [6]. This value is in good agreement, via the Clapeyron equation, with the observed slope of the $(P, T)$ equilibrium line at atmospheric pressure. The large increase in the entropy in the $\mathrm{N}$ phase, which helps to stabilize this phase, is not due to disorder resulting from thermal CT excitations but rather of vibrational origin. This is consistent with the observed systematic hardening of lattice-phonon modes in the I phase [20], due to closer interstack contacts (volume contraction) [13]. 


\section{Conclusion}

The exceptional behaviour of mixed-stack CT crystals around the N-I transition, where the cooperativity between self-trapped CT excitons is carried to extremes, gives the opportunity to study new states of condensed CT excitations such as liquid-like and crystal-like ones. To our knowledge such a $(P, T)$ phase diagram, analogous to the solid-liquid-gas one, has not been observed in any solid-state system before. Although the discussed results only concern the prototype compound, TTF-CA, many physical properties can be generalized to related compounds, with obviously some distinctive features. The liquid-gas-like line may be more or less extended and even may vanish in some cases. We can underline the case of $\mathrm{TTeC}_{1} \mathrm{TTF}$ [tetrakis (methyltelluro) tetrathiafulvalene]-TCNQ (tetracyanoquinodimethane), where the observed order-disorder type phase transition associated with an ordering of I dimers [32] is consistent with a liquid-solid-like transition. The nature of the ordering may be of different types: ferroelectric, antiferroelectric (cell-doubling) or more complex. Particularly interesting is the possibility of long-period structures and staging effects due to the frustration of interstack Coulomb interactions caused by crystalline arrangement. This probably occurs in TMB (tetramethylbenzidine)-TCNQ [34] and DMTTF (dimethyltetrathiafulvalene)-CA [35]. However, these different compounds have been investigated essentially on the basis of electronic and optical properties, and it will be important to perform precise structural studies in the future. Finally, we hope that a deep knowledge of the equilibrium N-I phase transition will help to understand non-equilibrium photo-induced phase transformations [9].

\section{Acknowledgments}

The reader will find in references the name of many colleagues with whom we have cooperated during experiments. We would like to warmly acknowledge them. We also wish to thank all Japanese colleagues for interesting and stimulating discussions during visits in Japan. H. Cailleau, M.H. Lemée-Cailleau and T. Luty thank The Yamada Foundation, The Japanese Society for Promotion of Sciences, and The Taniguchi Foundation for supports. This work was part of the Polish-French joint project no. 6721 .

\section{References}

[1] C.F. Klingshirn, Semiconductor Optics, Springer-Verlag, Berlin 1997 and references therein.

[2] Y. Toyozawa, Solid State Commun. 84, 255 (1992); Y. Toyozawa, Acta Phys. Pol. $A$ 87, 47 (1995).

[3] Relaxations of Excited States and Photo-Induced Structural Phase Transitions, Ed. K. Nasu, Springer-Verlag, Berlin 1997 and references therein.

[4] T. Luty, in Ref. [3], p. 142.

[5] H. Cailleau, M. Le Cointe, M.H. Lemée-Cailleau, in Ref. [3], p. 133.

[6] M.H. Lemée-Cailleau, M. Le Cointe, H. Cailleau, T. Luty, F. Moussa, J. Roos, D. Brinkmann, B. Toudic, C. Ayache, N. Karl, Phys. Rev. Lett. 79, 1690 (1997).

[7] J.B. Torrance, J.E. Vasquez, J.J. Mayerle, V.Y. Lee, Phys. Rev. Lett. 46, 253 (1981). 
[8] N. Nagaosa, J. Phys. Soc. Jpn. 55, 2754 (1986).

[9] S. Koshihara, Y. Tokura, T. Mitani, G. Saito, T. Koda, Phys. Rev. B 42, 6853 (1990); S. Koshihara, Y. Tokura, N. Sarukura, V. Segawa, T. Koda, K. Takeda, Synth. Met. 70, 1225 (1995).

[10] Y. Tokura, H. Okamoto, T. Koda, T. Mitani, G. Saito, Phys. Rev. B 38, 2215 (1988).

[11] J.J. Mayerle, J.B. Torrance, J.L. Crowley, Acta Crystallogr. B 35, 2988 (1979).

[12] C.S. Jacobsen, J.B. Torrance, J. Chem. Phys. 78, 112 (1983); A. Girlando, F. Marzola, C. Pecile, J.B. Torrance, J. Chem. Phys. 79, 1075 (1983); Y. Tokura, Y. Kaneko, H. Okamoto, S. Tanuma, T. Koda, T. Mitani, G. Saito, Mol. Cryst. Liq. Cryst. 125, 199 (1985).

[13] M. Le Cointe, M.H. Lemée-Cailleau, H. Cailleau, B. Toudic, L. Toupet, G. Heger, F. Moussa, P. Schweiss, K.H. Kraft, N. Karl, Phys. Rev. B 51, 3374 (1995).

[14] J. Gallier, B. Toudic, Y. Délugeard, H. Cailleau, M. Gourdji, A. Péneau, L. Guibé, Phys. Rev. B 47, 11688 (1993); M. Le Cointe, J. Gallier, H. Cailleau, M. Gourdji, A. Péneau, L. Guibé, Solid State Commun. 94, 455 (1995).

[15] T. Mitani, Y. Kaneko, S. Tanuma, Y. Tokura, T. Koda, G. Saito, Phys. Rev. B 35, 427 (1987).

[16] Y. Kaneko, S. Tanuma, Y. Tokura, T. Koda, T. Mitani, G. Saito, Phys. Rev. B 35, 8024 (1987).

[17] K. Takaoka, Y. Kaneko, H. Okamoto, Y. Tokura, T. Koda, T. Mitani, G. Saito, Phys. Rev. B 36, 3884 (1987).

[18] H. Hanfland, A. Brillante, A. Girlando, K. Syassen, Phys. Rev. B 38, 1456 (1988).

[19] H. Okamoto, T. Koda, Y. Tokura, T. Mitani, G. Saito, Phys. Rev. B 39, 10693 (1989).

[20] A. Moréac, A. Girard, Y. Délugeard, J. Phys., Condens. Matter 8, 3569 (1996).

[21] N. Nagaosa, J. Takimoto, J. Phys. Soc. Jpn. 55, 2735 (1986); 55, 2745 (1986); N. Nagaosa, J. Phys. Soc. Jpn. 55, 2754 (1986); M. Avignon, C.A. Balseiro, C.R. Proetto, B. Alascio, Phys. Rev. B 33, 205 (1986); B. Horovitz, J. Salyem, Phys. Rev. B 35, 7081 (1987); T. Luty, B. Kuchta, Phys. Rev. B 35, 8542 (1987); E.R. Gagliano, C.A. Balseiro, B. Alascio, Phys. Rev. B 37, 5697 (1988); A. Painelli, A. Girlando, Phys. Rev. B 37, 5748 (1988); T. Luty, Acta Phys. Pol. A 87, 1009 (1995); T. Iizuka-Sakano, T. Toyazawa, J. Phys. Soc. Jpn. 65, 671 (1996).

[22] C. Katan, P.E. Blöchl, P. Marge, C. Koenig, Phys. Rev. B 53, 12112 (1996);

C. Katan, C. Koenig, P.E. Blöchl, Solid State Commun. 102, 589 (1997).

[23] N. Nagaosa, J. Phys. Soc. Jpn. 55, 3488 (1986).

[24] N. Kojyo, Y. Onodera, J. Phys. Soc. Jpn. 56, 3228 (1983).

[25] K. Nasu, in Ref. [3], p. 3.

[26] M.J. Rice, E.J. Mele, Phys. Rev. Lelt. 49, 1455 (1982).

[27] M. Blume, V.J. Emery, R.B. Griffiths, Phys. Rev. A 4, 1071 (1971).

[28] J. Lajzerowicz, J. Sivardière, Phys. Rev. A 11, 2079 (1975).

[29] A. Morèac, A. Girard, Y. Dèlugeard, Y. Marqueton, J. Phys., Condens. Matter. 8, $3553(1996)$.

[30] R.M. Metzger, J.B. Torrance, J. Am. Chem. Soc. 107, 117 (1985).

[31] A. Girlando, A. Painelli, C. Pecile, G. Calestani, C. Rizzoli, R.M. Metzger, J. Chem. Phys. 98, 7692 (1993). 
[32] Y. Tokura, S. Koshihara, Y. Iwasa, H. Okamoto, T. Kamatsu, T. Koda, N. Iwasawa, G. Saito, Phys. Rev. Lett. 63, 2405 (1989).

[33] H. Okamoto, T. Mitani, Y. Tokura, S. Koshihara, T. Kamatsu, Y. Iwasa, T. Koda, G. Saito, Phys. Rev. B 43, 8224 (1991).

[34] Y. Iwasa, W. Watanabe, T. Koda, G. Saito, Phys. Rev. B 47, 2920 (1993).

[35] S. Aoki, T. Nakayama, A. Miura, Phys. Rev. B 48, 626 (1993). 